\title{
The Effect of Mindfulness Training On Resting-State Networks In Pre-Adolescent Children With Sub- Clinical Anxiety Related Attention Impairments.
}

Michelle F. Kennedy ( $\nabla$ michelle.kennedy@research.usc.edu.au )

University of the Sunshine Coast https://orcid.org/0000-0003-2095-6782

\section{Abdalla Z Mohamed}

University of the Sunshine Coast

\section{Paul Schwenn}

University of the Sunshine Coast

Denise Beaudequin

University of the Sunshine Coast

\section{Zack Shan}

University of the Sunshine Coast

\section{Daniel F. Hermens}

University of the Sunshine Coast

Jim Lagopoulos

University of the Sunshine Coast

\section{Research Article}

Keywords: fMRI, anxiety, attention, mindfulness, children.

Posted Date: December 3rd, 2021

DOI: https://doi.org/10.21203/rs.3.rs-1105420/v1

License: (a) This work is licensed under a Creative Commons Attribution 4.0 International License. Read Full License 


\section{Abstract}

Background: Mindfulness training has been associated with improved attention and affect regulation in preadolescent children with anxiety related attention impairments, however little is known about the underlying neurobiology. This study sought to investigate the impact of mindfulness training on functional connectivity of attention and limbic brain networks in pre-adolescents.

Methods: A total of 47 children (aged 9-11 years) participated in a 10-week mindfulness intervention. Anxiety and attention measures and resting-state fMRI were completed at pre-and post-intervention. Sustained attention was measured using the Conners Continuous Performance Test, while the anxiety levels were measured using the Spence Children's Anxiety Scale. Functional networks were estimated using independent-component analysis, and voxel-based analysis was used to determine the difference between the time-points to identify the effect of the intervention on the functional connectivity.

Results: There was a significant decrease in anxiety symptoms and improvement in attention scores following the intervention. From a network perspective, the results showed increased functional connectivity post intervention in the salience and fronto-parietal networks as well as the medial-inferior temporal component of the default mode network. Positive correlations were identified in the frontoparietal network with Hit Response Time and the Spence Children's Anxiety Scale total and between the default mode network and Hit Response Time.

Conclusions: A 10-week mindfulness intervention in children was associated with a reduction in anxiety related attention impairments, which were underpinned by concomitant changes in functional connectivity.

\section{Introduction}

Attention, involving the concentration of one's awareness on a given task to the exclusion of distracting stimuli, is often compromised in anxiety states (Petersen \& Posner, 2012). Specifically, emotion regulation requires cognitive control to minimize attention shifts to irrelevant stimuli, as dysfunction in these processes can produce deficits in inhibitory control (Eysenck, Derakshan, Santos, \& Calvo, 2007). Cognitive control and emotion regulation processes develop throughout childhood, and disruption to maturing brain networks during this period may contribute to dysfunction in these processes (Davidson, Amso, Anderson, \& Diamond, 2006; Luna, Garver, Urban, Lazar, \& Sweeney, 2004).

Mindfulness interventions have been associated with altered functional connectivity (FC) (BrefczynskiLewis, Lutz, Schaefer, Levinson, \& Davidson, 2007; Jha, Krompinger, \& Baime, 2007) in resting-state networks (V. Menon, 2015; Sha, Wager, Mechelli, \& He, 2019) and have been shown to improve attention and symptoms of anxiety in adult populations (Hofmann, Sawyer, Witt, \& Oh, 2010). Changes in FC in these networks can be measured at rest using resting-state functional magnetic resonance imaging (rsfMRI) (Raichle et al., 2001; Tang, Hölzel, \& Posner, 2015). Mindfulness studies investigating FC in resting-state networks in children are limited (Bauer et al., 2020; Marusak et al., 2018), with most 
focusing on clinical disorders or the efficacy of school-based mindfulness programs (Dunning et al., 2019; Flook et al., 2010). There is limited neurobiologically-informed research investigating the efficacy of mindfulness training on subclinical anxiety-related attention impairments in children.

The current study aimed to investigate the effect of mindfulness training on anxiety-related attention impairments and FC following a 10-week mindfulness intervention in preadolescent children. We hypothesized that mindfulness training would decrease anxiety and improve attention and would be associated with changes in FC within the networks that sub-serve these domains.

\section{Methods}

\subsection{Participants}

Children aged $9-11$ years of age, experiencing symptoms of anxiety and/or inattention, and proficient in spoken and written English were included in the study. Children with formal diagnoses of anxiety, attention, or autism spectrum disorder were excluded from the study as the focus was on sub-clinical symptomatology. Children with neurological disorders (e.g. epilepsy), intellectual disability, major medical illness, or who had sustained head injury (with loss of consciousness exceeding 10 minutes) were also excluded. Of $N=82$ expressions of interest received, $N=49$ met inclusion criteria. Two children withdrew during the study, thus $N=47$ participants completed the intervention and pre- and post-intervention assessments.

\subsection{Procedures}

Participants undertook the following assessments with a total duration of 60 minutes: (i) the Spence Children's Anxiety Scale (SCAS), (ii) the Conners Continuous Performance Test 3rd edition (CPT 3) and (iii) magnetic resonance imaging (MRI) scanning. Assessments were conducted at two time-points: (i) pre-intervention ('pre'); up to two weeks before the 10-week mindfulness intervention; and (ii) postintervention ('post'); up to two weeks following the mindfulness intervention. The study was conducted at the Thompson Institute, University of the Sunshine Coast, between July 2019 and January 2020.

\subsubsection{The Spence Children's Anxiety Scale (SCAS)}

The SCAS assessment (Spence, 1998) was used to provide an overall self-report measure of anxiety symptoms, as well as six childhood anxiety disorder subscale scores, in accordance with DSM- 5 criteria. The participants rated 38 statements relating to their fears, worries, and somatic symptoms using a Likert scale ranging from 0 (never) to 3 (always), with a possible score ranging from 0 - 114 .

\subsubsection{The Conners Continuous Performance Test $3^{\text {rd }}$ edition (CPT 3)}


The CPT 3 is a computerized test of attention and attention related problems in children 8-18 years (Conners, Epstein, Angold, \& Klaric, 2003). The CPT 3 measured four aspects of attention including sustained attention, inattentiveness, impulsivity and vigilance, via 360 trials presented in 14 minutes. Variables measured included detectability, errors of commissions, omissions and hit response time.

\subsubsection{Mindfulness Intervention}

The Mindfulness in Schools Project .b course is a school-based mindfulness curriculum, selected for demonstrated improvements in psychological well-being and cognitive skills post-intervention (Kuyken et al., 2013; Wilde et al., 2019). The course was delivered over ten 50min weekly sessions by a mindfulnesstrained researcher with .b curriculum certification (MK). Group sizes varied from $N=6$ to $N=12$.

Participants were required to practice the weekly mindfulness strategies supported by online videos, 3-4 times per week.

\subsubsection{Magnetic Resonance Imaging}

Imaging was performed on a Siemens Skyra 3T scanner (Siemens, Erlangen) using a 64-channel head and neck coil at the Nola Thompson Centre of Advanced Imaging, Thompson Institute. The T1-weighted images were collected using magnetization prepared-rapid gradient echo sequence with TR/TE/flip angle $=2000 \mathrm{~ms} / 1.7 \mathrm{~ms} / 7^{0}$; matrix $=224 \times 224 \times 176$, and resolution $=1.027 \times 1.027 \times 1 \mathrm{~mm}$. The rsfMRI was acquired using EPI with TR/TE $=1600 \mathrm{~ms} / 30 \mathrm{~ms}$, matrix $=74 \times 74 \times 48$, resolution of $3.027 \times 3.027 \times 3.0$ $\mathrm{mm}$, and a total of 300 volumes.

\subsubsection{MRI data pre-processing}

Data were pre-processed and analyzed using FMRIB's Software Library (FSL 6.0.4) and SPM12 (Neuroimaging, 2020) and CONN Functional Connectivity Toolbox (NITRC, 2020)

All T1-weighted images were corrected for field bias using N4BiasFieldCorrection, then were segmented into grey matter, white matter, and cerebrospinal fluid using the SPM-DARTEL pipeline. The pipeline was used to generate a study-specific template and normalize structural data to the template using field warp and grey matter deformation maps.

The rsfMRI scans were corrected for slice timing (FSL-slice timer) and head motion (FSL-mcflirt). The rsfMRI data was skull-stripped (FSL-bet), resampled to isotropic resolution of $2 \mathrm{~mm}$, and co-registered linearly to corresponding T1 scans in the native space (FSL-flirt). Outlier volumes within individuals were detected using the outlier detection algorithm in CONN. To filter data, motion artefacts, outlier information and signals extracted from white matter and ventricles were regressed out. Data were spatially normalized to the study-specific template (Ashburner and Friston, 1999) using SPM12 field-deformation 
maps. The rsfMRI data were spatially smoothed using a $6 \mathrm{~mm}$ Gaussian kernel of full width at half maximum to reduce spatial noise, and were band-pass temporally filtered $(f=0.007-0.1 \mathrm{~Hz})$.

\subsubsection{Independent component analysis}

To estimate the different functional networks, a dual-regression approach was utilized to calculate temporally-concatenated probabilistic independent components (Beckmann et al., 2009, 1995), using the Multivariate Exploratory Linear Decomposition into Independent Components (MELODIC), with number of components limited to 30. Functional networks were identified based on known networks (Beckmann et al., 2005; Thomas Yeo et al., 2011), including anterior and posterior default mode network (DMN), medialinferior temporal component of DMN, salience network (SLN), and fronto-parietal network (FPN).

\subsection{Statistical Analyses}

\subsubsection{Behavioral analyses}

Shapiro-Wilk tests determined assumptions of normality were violated. Wilcoxon signed-rank tests were used to compare behavioral data (SCAS, CPT 3) at pre- and post- intervention for: (i) change in anxiety scores (SCAS total and sub-total scores); and (ii) change in attention scores (CPT3 detectability, commissions, omissions and hit response time). All statistical tests were conducted using SPSS (Corp, 2019). Statistical significance was set at $p<.05$.

\subsubsection{Functional connectivity (FC) analysis}

To explicate the effect of the mindfulness intervention on FC, voxel-based analysis of the different brain networks was performed to estimate differences between pre- and post-intervention FC. Differences were estimated by entering individual maps into a general linear model; paired t-tests were performed with permutation testing (FSL-randomize; 5000 permutations) (Nichols and Holmes, 2002). All statistical analyses were corrected for age and gender. Results were corrected for multiple comparisons with familywise error ( $p \leq 0.05$, cluster $>100$ voxels) (Nichols \& Holmes, 2002). In addition, we performed correlations between FC maps and cognitive variables SCAS total, detectability commissions and hit response time at pre- and post-intervention time-points (significance at $p \leq 0.05$ ). These results were also corrected for multiple comparisons using family-wise error.

\section{Results}

\subsection{Behavioral results}


For the behavioral data, $N=4$ participants were excluded due to having an outlier score ( $\geq 3 S D$ ) at either time-point, hence, the final sample analyzed was $N=42$ (M 10.24yrs $S D 1.0$; males $N=28$ and females $N=14)$.

\subsubsection{Impact of mindfulness on anxiety (SCAS scores)}

Results showed a significant difference between median pre- and post- intervention scores for total anxiety score, separation anxiety score and obsessive-compulsive anxiety score (Table 1). There was no significant difference in pre- and post-intervention scores for the variables generalized anxiety disorder, social phobia, panic, agoraphobia or physical injury fears.

Table 1

Wilcoxon signed rank tests and effect sizes for pre- and post- mindfulness intervention behavioural scores (anxiety and attention).

\begin{tabular}{|c|c|c|c|c|c|c|}
\hline Variable & $N$ & $\begin{array}{l}\text { Pre- } \\
\text { median }\end{array}$ & $\begin{array}{l}\text { Post- } \\
\text { median }\end{array}$ & $\begin{array}{l}\text { Effect size } \\
\hat{r}\end{array}$ & $\begin{array}{l}\text { Wilcoxon } \\
\text { (z) }\end{array}$ & $\begin{array}{l}P \\
\text { value }\end{array}$ \\
\hline Anxiety & 42 & 32 & 27 & 0.4 & -2.2 & .015 \\
\hline Total score & & 4 & 3 & 0.6 & -3.0 & .001 \\
\hline Separation Anxiety & & 7 & 6 & 0.6 & -3.0 & .001 \\
\hline Obsessive-Compulsive & & 7 & 6 & 0.1 & -0.5 & .294 \\
\hline GAD & & 5 & 5 & 0.2 & -1.3 & .095 \\
\hline Social Phobia & & 4 & 4 & 0.1 & -0.8 & .021 \\
\hline $\begin{array}{l}\text { Panic and } \\
\text { agoraphobia }\end{array}$ & & 3 & 3 & 0.1 & -0.8 & .224 \\
\hline \multicolumn{7}{|l|}{ Physical injury fears } \\
\hline Attention & 42 & 47 & 34 & 0.5 & -2.7 & .003 \\
\hline Commissions & & -2 & -2 & 0.0 & -0.1 & .478 \\
\hline Omissions & & 492 & 494 & 0.2 & -1.6 & .053 \\
\hline HRT & & -2 & -2 & 0.2 & -1.6 & .053 \\
\hline \multicolumn{7}{|l|}{ Detectability } \\
\hline \multicolumn{7}{|c|}{ ^effect size calculated as $r=\frac{Z}{\sqrt{N}}$} \\
\hline \#one-tailed & & & & & & \\
\hline
\end{tabular}

\subsubsection{Impact of mindfulness on attention (CPT 3 scores)}


Participants performed better after the intervention, with results indicating a significant decrease in commission errors from pre- to post- intervention time-points (Table 1). There was no significant difference in pre- to post-intervention scores for the variables DPR, omissions or HRT.

\subsection{Neuroimaging results}

Five participants were excluded from the rsfMRI analysis due to incomplete data acquisition resulting from image distortion artefact, thus $N=42$ participants completed scans at both pre- and post-intervention time-points. Post-intervention, the group showed changes in FC at several networks including the SLN, FPN and the Medial-Inferior Temporal component (MTG) of the DMN and were corrected for multiple comparisons $(p \leq 0.05)$. However, no significant differences were observed in anterior or posterior components of the DMN.

For the SLN, compared to pre-intervention, there was increased FC post-intervention in multiple locations, as shown in Figure 1.

Similarly, the FPN showed increased FC post-intervention in the dIPFC, dmPFC, vmPFC, insula, middle frontal gyrus (MFG), inferior frontal gyrus (IFG), middle cingulate cortex (MCC), primary sensory and motor cortices and supplementary motor area as shown in Figure 2A. Similarly, the MTG of the DMN showed increased FC post-intervention in the dmPFC, ACC, IFG, MFG and superior frontal gyrus (SFG) as shown in Figure 2B.

\subsection{Correlational analysis}

There were $N=38$ individuals with complete behavioral and MRI data at both time-points, hence, correlational analyses were conducted at the pre-intervention time-point. The HRT had a positive correlation with right FPN connectivity to left amygdala $(d f=36, r=0.73 ; p<0.001)$, right hippocampus $(d f=36, r=0.47 ; p<0.001)$, caudate head $(d f=36, r=0.48 ; p \leq 0.05)$, left superior temporal gyrus $(d f=$ $36, r=0.49 ; p \leq 0.05)$, and precuneus $(d f=36, r=0.53 ; p \leq 0.05)$. In addition, SCAS total score was positively correlated with left FPN connectivity to right caudate head ( $d f=36, r=0.52 ; p \leq 0.05)$. Furthermore, the posterior DMN connectivity to right PCC $(d f=36, r=0.61 ; p \leq 0.05)$ and left PCC ( $d f=36$, $r=0.52, p \leq 0.05)$, and precuneus $(d f=36, r=0.49 ; p \leq 0.05)$ were positively correlated with the attention variable HRT. No correlations were observed between the behavioral measures and the salience FC. In contrast to the pre-intervention time-point, there were no significant correlations at the post-intervention time-point for any of the functional networks.

\section{Discussion}

This is the first study to investigate effects of a mindfulness intervention on FC in resting-state networks in a preadolescent cohort with sub-clinical anxiety-related attention impairments. Understanding mindfulness-induced neural and associated behavioral changes is crucial to support the efficacy of early interventions (Sylvester et al., 2012). Our results showed increased FC in the resting-state networks investigated [SLN, FPN, and DMN (MTG)] following the mindfulness intervention. Increased FC was 
observed in the SLN and FPN, suggesting improvements in cognitive control and emotion regulation, however increased FC in the DMN in contrast to decreased FC in the DMN observed in adult studies may be associated with participants' maturing brain networks (Brewer et al., 2011; Hasenkamp, WilsonMendenhall, Duncan, \& Barsalou, 2012; Marusak et al., 2018). These observed changes in FC were associated with improved behavioral outcomes. Positive correlations were identified pre-intervention in the FPN to several brain regions for the HRT and the SCAS total and in the DMN to several brain regions for the HRT. The SLN, including key nodes insula and ACC (Uddin, 2015), is involved in the detection, filtering and orientation to salient stimuli (Vinod Menon \& Uddin, 2010). Decreased FC in the SLN to the prefrontal regions and insula has been implicated in deficits in emotion regulation and cognitive control, processes recognized as underlying anxiety and attention disorders (Etkin, Egner, \& Kalisch, 2011; Uddin, Supekar, Ryali, \& Menon, 2011). Recent research has associated mindfulness interventions with increased FC in the SLN to the insula, vmPFC, dmPFC, dIPFC, ACC, hippocampus and amygdala and improvements in anxiety symptoms (Marusak et al., 2018; Tang et al., 2015), consistent with our findings. Specifically, increased FC in the prefrontal regions has been associated with improved cognitive control required for effective processing of emotionally salient stimuli (Etkin et al., 2011; Kober et al., 2008). Furthermore, our observation of increased SLN connectivity to the dIPFC, hippocampus and amygdala has been associated with improvements in switching attention from emotionally salient events. (Forster, Nunez Elizalde, Castle, \& Bishop, 2015; Zheng et al., 2017). The FPN is the central executive network, and with frontal regions, supports executive control of attention (Corbetta \& Shulman, 2002; Petersen \& Posner, 2012). Decreased FC in the FPN is associated with deficits in executive function, specifically cognitive control and emotion regulation and has been reported in children with impairments in attention and anxiety (Rosenberg et al., 2016). Mindfulness training has been associated with increased FC in the FPN and increased cognitive control and emotion regulation in adult and youth populations (Hölzel et al., 2013; Vago \& David, 2012). Our findings indicate increased FC to the vmPFC, dIPFC, vmPFC, IFG, MFG, and insula in our cohort. The insula has been implicated in introspective thoughts (Critchley, Wiens, Rotshtein, Öhman, \& Dolan, 2004), thus increased FC to the dIPFC and vmPFC may modulate attention to anxious thoughts (Vinod Menon \& Uddin, 2010; Namkung, Kim, \& Sawa, 2017). Moreover, an overall decrease in participants' anxiety scores was supported by improved FC in these regions. Increased FC to the IFG and MFG has been implicated in increased response inhibition to irrelevant internal emotional stimuli, thus supporting sustained attention (Japee, Holiday, Satyshur, Mukai, \& Ungerleider, 2015; Petersen \& Posner, 2012). Furthermore, an overall decrease in participants' commission scores supports improvement in inhibitory control. Attention to self-referential thoughts or 'mind-wandering' has been associated with increased activation in the DMN during rsfMRI, as well as anxiety and attention issues (Brewer et al., 2011; Marusak et al., 2018). Mindfulness training has been shown to decrease activation in the DMN in adults, thus decreasing mind-wandering (Jha et al., 2007). Our result of increased FC within the DMN regions of dmPFC, ACC, IFG, MFG, and SFG may underpin improvements in emotion regulation and corroborate recent findings by (Hafeman et al., 2020). However, our findings are in contrast with another study's findings of decreased FC between the DMN and dIPFC (Bauer et al., 2020). Previous studies established that developmental FC changes in children are characterized by weakening shortrange FC and strengthening long-range FC (Kelly et al., 2009; Uddin, Supekar, \& Menon, 2010). Our 
findings of increased FC were observed after a relatively short period of 10-12 weeks between MRI scans. Taken together, we suggest that FC changes within networks in this study are due to the mindfulness intervention, and less likely associated with developmental changes. Furthermore, increased FC between the SLN, FPN and DMN following the mindfulness intervention may indicate improved cognitive control and emotion regulation (Marusak et al., 2018). The SLN plays a crucial role in switching activation between the FPN and DMN, as these networks support processing the emotional salience of irrelevant stimuli and are often dysfunctional in children with anxiety-related attention impairments (Sha et al., 2019). At pre-intervention, the right FPN was correlated with slower response times, often associated with inattentiveness. Specifically, increased FC to the left amygdala and right hippocampus sends direct projections to the caudate, negatively affecting goal-directed behavior (Grahn, Parkinson, \& Owen, 2008). The FPN connectivity to the caudate showed a positive correlation with SCAS total score, indicating increased FC is associated with elevated anxiety symptoms. Correspondingly, greater caudate activation has been reported in children with high levels of behavioral inhibition, considered a risk factor for anxiety (Lahat, Benson, Pine, Fox, \& Ernst, 2018; Vincent, Kahn, Snyder, Raichle, \& Buckner, 2008). However, this response was improved in our participants following the mindfulness intervention, with no significant correlations observed between the FPN FC and the HRT or total SCAS score, suggesting normalization of participants' FC. Momentary lapses of attention have been associated with increased activity in the DMN wherein strong interactivity of key nodes PCC/precuneus, may be linked to slower HRT (Cavanna \& Trimble, 2006; Weissman, Roberts, Visscher, \& Woldorff, 2006). Moreover, the correlation of DMN connectivity correlation with HRT might arise from increased self-awareness and reduced task performance, which is known to take place in participants with behavioral deficits (Alexopoulos et al., 2012; Zhang \& Li, 2012). At post-intervention, these observed correlations of the DMN connectivity were not significant, suggesting the mindfulness intervention may have improved FC and behavioral performance (Brewer et al., 2011). Taken together, the lack of correlations post-intervention in the FPN and DMN networks suggests the mindfulness intervention altered FC in these networks and reduced behavioral scores. Thus, the observed pre-intervention correlations and the behavioral variables may be markers for anxiety-related attention impairments in pre-adolescent populations. Our study has several limitations that require mentioning. Firstly, a control group was not included in the design, as sub-clinical anxiety is pervasive in preadolescent children, with approximately up to $70 \%$ of children reporting they worry "every now and then" (Cartwright-Hatton, 2006; Muris, Merckelbach, Meesters, \& van den Brand, 2002). Secondly a mindfulness scale was not included, as the concepts of metacognition and interoceptive awareness are still developing during pre-adolescence; thus, researcher involvement to clarify questions may have increased risk of response bias. Finally, as this was a group program, some improvements may be attributed to social interactions and group discussions.

\section{Conclusions}

Our study has shown that mindfulness was associated with improved anxiety and attention behavioural scores as well as altered FC in several interconnected resting-state networks. Furthemore, the findings of positive correlations at pre-intervention in the FPN and DMN networks to several brain regions with the 
attention variable HRT, and the anxiety variable SCAS total score, suggests that excessive FC in these networks may be a marker of anxiety-related attention impairments in sub-clinical populations. Our findings suggest mindfulness strategies may be an effective intervention for sub-clinical anxiety-related attention impairments in pre-adolescent children.

\section{Declarations}

6.1 Funding Not applicable

6.2 Conflict of Interest The authors have no conflict of interest to declare

6.3 Ethics Approval The Combatting Anxiousness in Learning Minds (CALM) study protocol was approved by the Human Research Ethics Committee, University of the Sunshine Coast (\#S181226).

6.4 Consent to participate Informed, written consent was obtained from all participants and their caregivers prior to participation in the study.

6.5 Consent for publication not applicable; no identifiable information has/will be published

6.6 Availability of data and material the data will be shared upon request from the corresponding author.

6.7 Code availability not applicable

6.8 Competing interests not applicable

6.9 Authors' contributions MK carried out recruitment of participants and the compilation of the database. MK, AM and PS, performed the statistical analyses. MK wrote the first draft of the manuscript. MK, AM, DB, ZS, DH and JL helped write parts of the manuscript. DH and JL supervised the entire study. All authors had full access to all study data and analyses, participated in preparing this manuscript, and approved its final, submitted form.

\section{References}

1. Alexopoulos, G. S., Hoptman, M. J., Kanellopoulos, D., Murphy, C. F., Lim, K. O., \& Gunning, F. M. (2012). Functional connectivity in the cognitive control network and the default mode network in latelife depression. Journal of Affective Disorders, 139(1), 56-65. doi:https://doi.org/10.1016/j.jad.2011.12.002

2. Bauer, C. C. C., Rozenkrantz, L., Caballero, C., Nieto-Castanon, A., Scherer, E., West, M. R., \& WhitfieldGabrieli, S. (2020). Mindfulness training preserves sustained attention and resting state anticorrelation between default-mode network and dorsolateral prefrontal cortex: A randomized controlled trial. Human brain mapping, 41(18), 5356-5369

3. Brefczynski-Lewis, J. A., Lutz, A., Schaefer, H. S., Levinson, D. B., \& Davidson, R. J. (2007). Neural correlates of attentional expertise in long-term meditation practitioners. Proceedings of the National 
Academy of Sciences, 104(27), 11483-11488

4. Brewer, J. A., Worhunsky, P. D., Gray, J. R., Tang, Y. Y., Weber, J., \& Kober, H. (2011). Meditation experience is associated with differences in default mode network activity and connectivity. Proceedings of the National Academy of Sciences, 108(50), 20254. doi:10.1073/pnas.1112029108

5. Cartwright-Hatton, S. (2006). Worry in childhood and adolescence. Worry and its psychological disorders: Theory, assessment and treatment, 81-97

6. Cavanna, A. E., \& Trimble, M. R. (2006). The precuneus: a review of its functional anatomy and behavioural correlates. Brain, 129(3), 564-583. doi:10.1093/brain/awl004

7. Conners, C. K., Epstein, J. N., Angold, A., \& Klaric, J. (2003). Continuous performance test performance in a normative epidemiological sample. Journal of Abnormal Child Psychology, 31(5), 555-562

8. Corbetta, M., \& Shulman, G. L. (2002). Control of goal-directed and stimulus-driven attention in the brain. Nature reviews neuroscience, 3(3), 201-215

9. Corp, I. (2019). IBM SPSS Statistics for Windows (Version 26). Armonk NY: IBM Corp. Retrieved from https://www.ibm.com/au-en/products/spss-statistics

10. Critchley, H. D., Wiens, S., Rotshtein, P., Öhman, A., \& Dolan, R. J. (2004). Neural systems supporting interoceptive awareness. Nature neuroscience, 7(2), 189-195

11. Davidson, M. C., Amso, D., Anderson, L. C., \& Diamond, A. (2006). Development of cognitive control and executive functions from 4 to 13 years: Evidence from manipulations of memory, inhibition, and task switching. Neuropsychologia, 44(11), 2037-2078

12. Dunning, D. L., Griffiths, K., Kuyken, W., Crane, C., Foulkes, L., Parker, J., \& Dalgleish, T. (2019). Research Review: The effects of mindfulness-based interventions on cognition and mental health in children and adolescents - a meta-analysis of randomized controlled trials. Journal of Child Psychology and Psychiatry, 60(3), 244-258. doi:https://doi.org/10.1111/jcpp.12980

13. Etkin, A., Egner, T., \& Kalisch, R. (2011). Emotional processing in anterior cingulate and medial prefrontal cortex. Trends in Cognitive Sciences, 15(2), 85-93

14. Eysenck, M. W., Derakshan, N., Santos, R., \& Calvo, M. G. (2007). Anxiety and cognitive performance: attentional control theory. Emotion, 7(2), 336

15. Flook, L., Smalley, S. L., Kitil, M. J., Galla, B. M., Kaiser-Greenland, S., Locke, J., \& Kasari, C. (2010). Effects of mindful awareness practices on executive functions in elementary school children. Journal of applied school psychology

16. Forster, S., Nunez Elizalde, A. O., Castle, E., \& Bishop, S. J. (2015). Unraveling the anxious mind: anxiety, worry, and frontal engagement in sustained attention versus off-task processing. Cerebral Cortex, 25(3), 609-618

17. Grahn, J. A., Parkinson, J. A., \& Owen, A. M. (2008). The cognitive functions of the caudate nucleus. Progress in neurobiology, 86(3), 141-155. doi:https://doi.org/10.1016/j.pneurobio.2008.09.004 
18. Hasenkamp, W., Wilson-Mendenhall, C. D., Duncan, E., \& Barsalou, L. W. (2012). Mind wandering and attention during focused meditation: a fine-grained temporal analysis of fluctuating cognitive states. Neurolmage, 59(1), 750-760

19. Hofmann, S. G., Sawyer, A. T., Witt, A. A., \& Oh, D. (2010). The effect of mindfulness-based therapy on anxiety and depression: A meta-analytic review. Journal of consulting and clinical psychology, 78(2), 169

20. Hölzel, B. K., Hoge, E. A., Greve, D. N., Gard, T., Creswell, J. D., Brown, K. W., \& Lazar, S. W. (2013). Neural mechanisms of symptom improvements in generalized anxiety disorder following mindfulness training. Neurolmage: Clinical, 2, 448-458

21. Japee, S., Holiday, K., Satyshur, M. D., Mukai, I., \& Ungerleider, L. G. (2015). A role of right middle frontal gyrus in reorienting of attention: a case study. Frontiers in systems neuroscience, 9, 23. doi:https://doi.org/10.3389/fnsys.2015.00023

22. Jha, A. P., Krompinger, J., \& Baime, M. J. (2007). Mindfulness training modifies subsystems of attention. Cognitive, Affective, \& Behavioral Neuroscience, 7(2), 109-119

23. Kelly, A. M. C., Di Martino, A., Uddin, L. Q., Shehzad, Z., Gee, D. G., Reiss, P. T., \& Milham, M. P. (2009). Development of anterior cingulate functional connectivity from late childhood to early adulthood. Cerebral Cortex, 19(3), 640-657

24. Kober, H., Barrett, L. F., Joseph, J., Bliss-Moreau, E., Lindquist, K., \& Wager, T. D. (2008). Functional grouping and cortical-subcortical interactions in emotion: a meta-analysis of neuroimaging studies. Neurolmage, 42(2), 998-1031

25. Kuyken, W., Weare, K., Ukoumunne, O. C., Vicary, R., Motton, N., Burnett, R., \& Huppert, F. (2013). Effectiveness of the Mindfulness in Schools Programme: non-randomised controlled feasibility study. The British Journal of Psychiatry, 203(2), 126-131

26. Lahat, A., Benson, B. E., Pine, D. S., Fox, N. A., \& Ernst, M. (2018). Neural responses to reward in childhood: relations to early behavioral inhibition and social anxiety. Social cognitive and affective neuroscience, 13(3), 281-289. doi:10.1093/scan/nsw122

27. Luna, B., Garver, K. E., Urban, T. A., Lazar, N. A., \& Sweeney, J. A. (2004). Maturation of cognitive processes from late childhood to adulthood. Child Development, 75(5), 1357-1372

28. Marusak, H. A., Elrahal, F., Peters, C. A., Kundu, P., Lombardo, M. V., Calhoun, V. D., \& Rabinak, C. A. (2018). Mindfulness and dynamic functional neural connectivity in children and adolescents. Behavioural brain research, 336, 211-218. doi:https://doi.org/10.1016/j.bbr.2017.09.010

29. Menon, V. (2015). Large-scale functional brain organization. Brain mapping,449-459

30. Menon, V., \& Uddin, L. Q. (2010). Saliency, switching, attention and control: a network model of insula function. Brain Structure and Function, 214(5-6), 655-667

31. Muris, P., Merckelbach, H., Meesters, C., \& van den Brand, K. (2002). Cognitive development and worry in normal children. Cognitive Therapy and Research, 26(6), 775-787

32. Namkung, H., Kim, S. H., \& Sawa, A. (2017). The insula: an underestimated brain area in clinical neuroscience, psychiatry, and neurology. Trends in neurosciences, 40(4), 200-207 
33. Neuroimaging, W. C., \& f., H. (2020). SPM12. London, United Kingdom. Retrieved from https://www.fil.ion.ucl.ac.uk/spm/software/spm12/

34. Nichols, T. E., \& Holmes, A. P. (2002). Nonparametric permutation tests for functional neuroimaging: A primer with examples. Human brain mapping, 15(1), 1-25. doi:https://doi.org/10.1002/hbm.1058

35. NITRC (2020). CONN toolbox (Version 17). Retrieved from .org/projects/conn, RRID:SCR_009550

36. Petersen, S. E., \& Posner, M. I. (2012). The attention system of the human brain: 20 years after. Annual review of neuroscience, 35, 73-89

37. Raichle, M. E., MacLeod, A. M., Snyder, A. Z., Powers, W. J., Gusnard, D. A., \& Shulman, G. L. (2001). A default mode of brain function. Proceedings of the National Academy of Sciences, 98(2), 676-682

38. Rosenberg, M. D., Finn, E. S., Scheinost, D., Papademetris, X., Shen, X., Constable, R. T., \& Chun, M. M. (2016). A neuromarker of sustained attention from whole-brain functional connectivity. Nature neuroscience, 19(1), 165-171

39. Sha, Z., Wager, T. D., Mechelli, A., \& He, Y. (2019). Common Dysfunction of Large-Scale Neurocognitive Networks Across Psychiatric Disorders. Biological Psychiatry, 85(5), 379-388. doi:https://doi.org/10.1016/j.biopsych.2018.11.011

40. Spence, S. H. (1998). A measure of anxiety symptoms among children. Behaviour Research and Therapy, 36(5), 545-566. doi:https://doi.org/10.1016/S0005-7967(98)00034-5

41. Sylvester, C. M., Corbetta, M., Raichle, M. E., Rodebaugh, T. L., Schlaggar, B. L., Sheline, Y. I., \& Lenze, E. J. (2012). Functional network dysfunction in anxiety and anxiety disorders. Trends in neurosciences, 35(9), 527-535. doi:https://doi.org/10.1016/j.tins.2012.04.012

42. Tang, Y. Y., Hölzel, B. K., \& Posner, M. I. (2015). The neuroscience of mindfulness meditation. Nature reviews neuroscience, 16(4), 213-225. doi:10.1038/nrn3916

43. Uddin, L. Q. (2015). Salience processing and insular cortical function and dysfunction. Nature reviews neuroscience, 16(1), 55-61

44. Uddin, L. Q., Supekar, K., \& Menon, V. (2010). Typical and atypical development of functional human brain networks: insights from resting-state FMRI. Frontiers in systems neuroscience, 4, 21

45. Uddin, L. Q., Supekar, K. S., Ryali, S., \& Menon, V. (2011). Dynamic reconfiguration of structural and functional connectivity across core neurocognitive brain networks with development. Journal of neuroscience, 31(50), 18578-18589

46. Vago, D. R., \& David, S. A. M. D. (2012). Self-awareness, self-regulation, and self-transcendence (SART): a framework for understanding the neurobiological mechanisms of mindfulness. Frontiers in Human Neuroscience, 6, 296

47. Vincent, J. L., Kahn, I., Snyder, A. Z., Raichle, M. E., \& Buckner, R. L. (2008). Evidence for a frontoparietal control system revealed by intrinsic functional connectivity. Journal of neurophysiology, 100(6), 3328-3342

48. Weissman, D. H., Roberts, K. C., Visscher, K. M., \& Woldorff, M. G. (2006). The neural bases of momentary lapses in attention. Nature neuroscience, 9(7), 971-978 
49. Wilde, S., Sonley, A., Crane, C., Ford, T., Raja, A., Robson, J., \& Kuyken, W. (2019). Mindfulness Training in UK Secondary Schools: a Multiple Case Study Approach to Identification of Cornerstones of Implementation. Mindfulness, 10(2), 376-389. doi:10.1007/s12671-018-0982-4

50. Zhang, S., \& Li, C. (2012). Task-Related, Low-Frequency Task-Residual, and Resting State Activity in the Default Mode Network Brain Regions. Frontiers in psychology, 3(172), doi:10.3389/fpsyg.2012.00172

51. Zheng, J., Anderson, K. L., Leal, S. L., Shestyuk, A., Gulsen, G., Mnatsakanyan, L., \& Knight, R. T. (2017). Amygdala-hippocampal dynamics during salient information processing. Nature communications, 8(1), 1-11

\section{Figures}

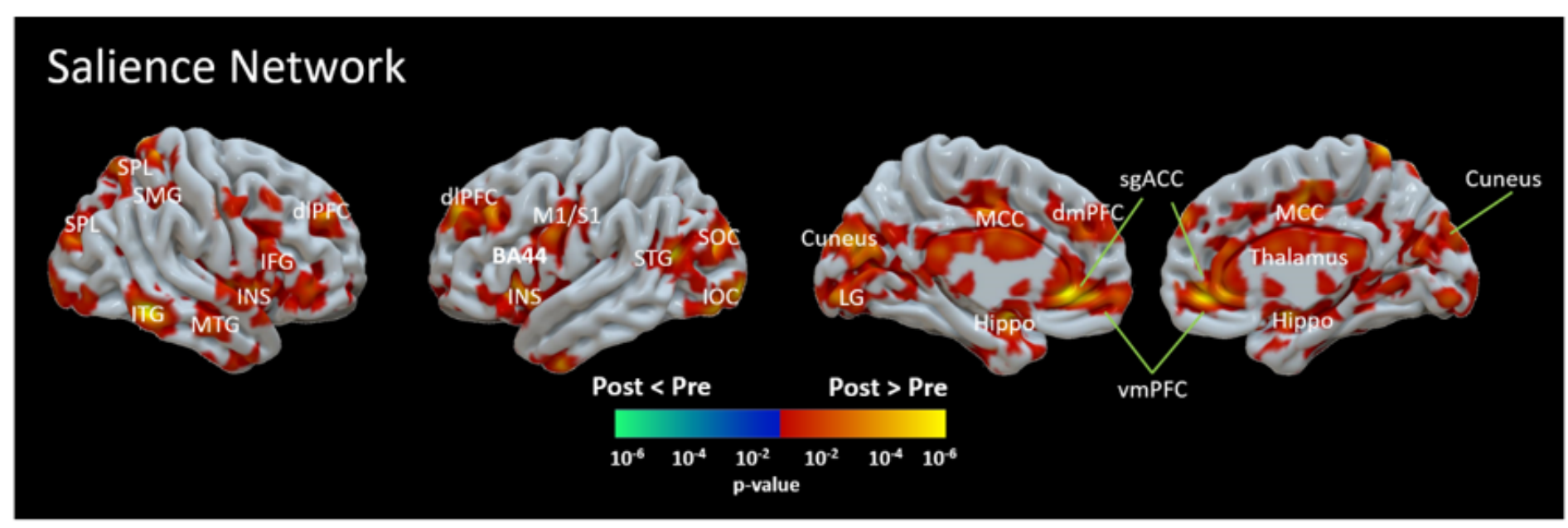

\section{Figure 1}

Differences between the pre- and post-intervention in functional connectivity (FC) of the salience network (SLN). The results showed increased FC of the SLN following the 10 week mindfulness intervention. The regions with increased FC of the SLN are linked to the default mode network (supra-marginal gyrus, ventromedial pre-frontal cortex -vmPFC, posterior cingulate cortex - PCC, amygdala and hippocampus, inferior temporal gyrus-ITG), visual perception (superior occipital gyrus, inferior occipital gyrus ), sensorimotor function (putamen, primary motor cortex and somatosensory cortex), cognitive functions (dorsolateral pre-frontal cortex - dIPFC, inferior frontal gyrus - IFG, superior parietal lobule), or attention (anterior cingulate cortex - ACC, mid-cingulate cortex and insula). 


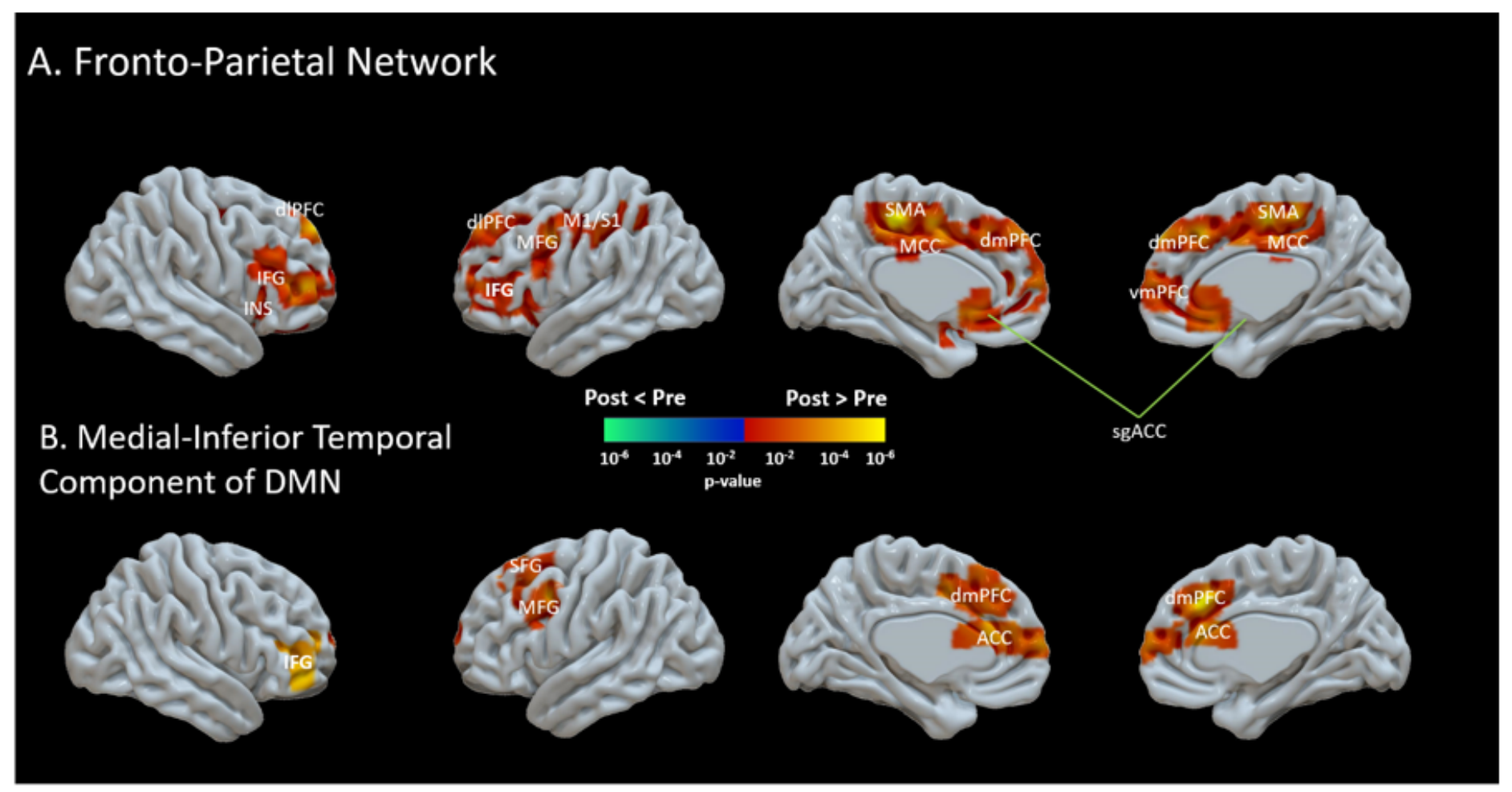

\section{Figure 2}

Differences between the pre- and post-intervention time-points in the functional connectivity (FC) of: (A) Frontal-Parietal network (FPN) and (B) the Medial-inferior temporal component (MTG) of the Default Mode Network (DMN). The results showed increased FC of the FPN and MTG following the 10-week mindfulness intervention. The regions with increased FC of the FPN are linked to the sensorimotor (primary sensory and motor cortices (S1, M1), supplementary motor area (SMA), middle frontal gyrus (MFG), cognitive and executive (dIPFC, dmPFC, vmPFC, inferior frontal gyrus (IFG)), and attention (insular cortex (INS), ACC). The regions with increased FC of the MTG of the DMN are linked to different brain functions including the dmPFC, ACC, IFG, MFG, superior frontal gyrus (SFG).

\section{Supplementary Files}

This is a list of supplementary files associated with this preprint. Click to download.

- BIBChecklist.docx 